\title{
Identificação de medicamentos "não apropriados para crianças" em prescrições de unidade de tratamento intensivo pediátrica
}

\author{
Prescription of drugs not appropriate for children in a Pediatric Intensive Care Unit \\ Paulo R.A. Carvalho ${ }^{1}$, Clarissa G. Carvalho ${ }^{2}$, Patrícia T. Alievi ${ }^{3}$, \\ Jaqueline Martinbiancho ${ }^{4}$, Eliana A. Trotta ${ }^{5}$
}

\section{Resumo}

Objetivo: Avaliar a extensão do uso de medicamentos não apropriados para crianças em prescrições de uma unidade de tratamento intensivo pediátrica (UTIP) terciária, de acordo com os padrões estabelecidos pela FDA.

Métodos: Estudo transversal, observacional, baseado na avaliação das prescrições de todos os pacientes admitidos na UTIP do Hospital de Clínicas de Porto Alegre, no período de seis semanas consecutivas. Foram considerados idade, sexo, peso, doença prévia, motivo de admissão na UTIP, e PIM (pediatric index of mortality) dos pacientes, e todos os medicamentos prescritos e suas indicações, com as respectivas apresentações, doses, frequiências e vias de administração. O critério de adequação na prescrição dos medicamentos seguiu a classificação de aprovação da FDA, baseada no catálogo de informações de medicamentos USP DI 2001, em três faixas etárias pediátricas.

Resultados: Os dados foram obtidos nos meses de julho e agosto de 2002, em diferentes dias, de seis semanas consecutivas, baseados nas prescrições de 51 pacientes em 54 admissões de UTIP. A mediana de idade dos pacientes foi 10,5 meses, sendo $61 \%$ do sexo masculino. Dois terços $(65 \%)$ dos pacientes apresentavam doença prévia, e $87 \%$ das admissões ocorreram por motivos clínicos, sendo $57 \%$ por causas respiratórias. Foram registrados 747 itens de prescrição, com prevalências de $10,5 \%$ para medicamentos não aprovados e $49,5 \%$ para medicamentos não padronizados. A distribuição das prevalências de prescrição, tanto pela faixa etária quanto pelo nível de gravidade dos pacientes na admissão (de acordo com as categorias de risco do PIM), nas três classes de medicamentos, não mostrou diferença estatística entre os grupos.

Conclusão: A alta prevalência de prescrições com medicamentos não apropriados para crianças confirma, também no nosso meio, o uso inadequado e inadvertido de medicamentos não testados ou de apresentações não padronizadas para crianças em UTIP. Isso aponta para a necessidade de estimular a realização de estudos sobre qualidade, eficácia e segurança de medicamentos para uso pediátrico.

J Pediatr (Rio J) 2003;79(5):397-402: Crianças, medicamentos, cuidado intensivo, prescrição de não aprovados, prescrição de não padronizados.

\begin{abstract}
Objective: To assess the extent of use of drugs not appropriate for children in prescriptions issued in a tertiary pediatric intensive care unit (PICU), according to FDA standards.

Methods: Observational cross-sectional study. The prescriptions issued to all patients admitted to the PICU at Hospital de Clínicas de Porto Alegre, Brazil, over a six-week period were assessed. Patients' age, sex, weight, prior disease, reason for admission to the PICU and pediatric index of mortality (PIM) were recorded, as were all drugs prescribed, their indications, presentations, doses, frequencies and means of administration. Adequacy for prescription of drugs in three pediatric age ranges was defined according to USA Food and Drug Administration (FDA) approval classification, based on the USP DI 2001 drug reference database.
\end{abstract}

Results: Data were obtained in the months of July and August 2002, on different days, for six consecutive weeks, based on prescriptions issued to 51 patients in 54 admissions to the PICU. Median patient age was 10.5 months; $61 \%$ of patients were male. Two thirds of patients $(65 \%)$ presented prior disease. $87 \%$ of admissions were due to clinical reasons, of which 57\% were respiratory complaints. A total of 747 prescription items were registered, with prevalence of $10.5 \%$ for nonapproved uses and $49.5 \%$ for off-label uses. No statistically significant difference was found in the distribution of prevalence of irregular prescription either by the three age ranges or by level of severity of disease at admission (according to PIM risk categories).

Conclusion: The high prevalence of prescription of drugs not appropriate for children confirms, in the Brazilian context, the inadequate and inadvertent use of drugs either not approved or offlabel for PICU use. This demonstrates the need to encourage further studies on the quality, efficacy and safety of drugs for pediatric use.

J Pediatr (Rio J) 2003;79(5):397-402: Children, drugs, intensive care, not approved prescribing, off label prescribing.

1. Doutor em Pediatria pela UFRGS. Professor Adjunto do Dep. de Pediatria da Universidade Federal do Rio Grande do Sul (UFRGS).

2. Doutoranda de graduação da Faculdade de Medicina da UFRGS.

3. Médica Residente de $3^{\circ}$ ano da UTI Pediátrica do Hospital de Clínicas de Porto Alegre (HCPA).

4. Farmacêutica do Serviço de Farmácia do HCPA.

5. Professora Adjunta do Dep. de Pediatria da UFRGS. Médica chefe da UTI Pediátrica do HCPA.

Artigo submetido em 27.02.03, aceito em 17.06.03. 


\section{Introdução}

Em decorrência de prováveis limitações éticas relacionadas à pesquisa de medicamentos envolvendo crianças em décadas passadas, mais de $50 \%$ dos medicamentos prescritos para pacientes pediátricos nos EUA não são aprovados ou padronizados para uso em crianças ${ }^{1}$. No nosso meio, supeita-se que a taxa de uso de medicamentos não apropriados para crianças, em pacientes pediátricos, também seja elevada, especialmente em nível hospitalar.

O conceito de medicamentos não apropriados para crianças envolve uma variedade de condições, gerando inclusive superposição das nomenclaturas relatadas ${ }^{2-8}$. Algumas das referências consultadas permitem concluir que o termo não aprovado (not approved) considera medicamentos não aprovados para uso como um todo, não aprovados para uso em crianças, contra-indicados para uso em crianças, manufaturados no hospital, modificados no hospital, ou sem dosagem específica para crianças ${ }^{2-4,8}$. O termo não padronizado (offlabel) considera medicamentos prescritos de forma diferente daquela orientada na bula, em relação à faixa etária, à dose, à frequiência, à apresentação, à via de administração ou à indicação para uso em crianças $^{2-4,8,9}$.

Em alguns países existem relatos de altas prevalências na utilização de medicamentos não aprovados ou não padronizados para crianças, tanto em consultórios pediátricos quanto em unidades de internação e de tratamento intensivo pediátricas ${ }^{2-10}$, de modo não justificado, ou mesmo por desconhecimento dos prescritores em relação a essas peculiaridades.

Com muita freqüência, a prescrição e o uso desses medicamentos, nas faixas pediátricas, são baseados em extrapolações de doses e/ou modificações de formulações para adultos, ignorando-se completamente as diferenças entre crianças e adultos, e submetendo aquelas aos riscos de eficácia não comprovada e de paraefeitos não avaliados ${ }^{1,11}$.

Há mais de 30 anos, Shirkey já reconhecia a existência do sério dilema da padronização de medicamentos pediátricos, chamando-os de "órfãos terapêuticos". Especialmente na última década, inúmeras iniciativas da Agência de Controle de Medicamentos e Alimentos (Food and Drug Administration - FDA) e do Comitê de Medicamentos da Academia Americana de Pediatria, ambas dos EUA, ambas com reconhecimento internacional, têm concorrido no sentido de estimular a pesquisa, aprovação e padronização de formulações medicamentosas para uso em crianças ${ }^{12}$.

O objetivo deste estudo foi avaliar a extensão do uso de medicamentos não apropriados para crianças em prescrições de uma unidade de tratamento intensivo pediátrica (UTIP) terciária, de acordo com os padrões estabelecidos pela FDA.

\section{Casuística e métodos}

Foi realizado estudo transversal, observacional, baseado na avaliação das prescrições dos pacientes admitidos na
UTIP do Hospital de Clínicas de Porto Alegre, no período de seis semanas consecutivas, considerando um dia diferente em cada semana avaliada. A população estudada foi constituída de todos os pacientes internados na UTIP em cada um dos dias da avaliação, durante todo o período do estudo, excluídos aqueles de permanência prolongada na UTI, e cuja prescrição já teria sido avaliada nas semanas anteriores. Não foram consideradas as prescrições de soluções cristalóides, nutrição parenteral, derivados de sangue e oxigênio.

As variáveis consideradas no estudo incluíram a idade dos pacientes, o sexo, o peso, a superfície corporal, a doença mórbida prévia, o motivo de admissão na UTIP, o $\mathrm{PIM}^{13}$ (pediatric index of mortality), todos os medicamentos prescritos e suas indicações, com as respectivas apresentações, doses, freqüências e vias de administração.

O critério de adequação na prescrição dos medicamentos adotado no estudo foi baseado em classificação de aprovação da FDA, tendo como referência o catálogo de informações de medicamentos USP DI $2001^{14}$. Não foi utilizado padrão nacional de adequação porque a ANVISA (Agência Nacional de Vigilância Sanitária), que regula o registro de medicamentos no Brasil, não possui catálogo semelhante ${ }^{15}$. Foram definidas três classes de medicamentos: (a) classe aprovados - medicamentos aprovados para uso em crianças, que incluiria todos os medicamentos prescritos que são aprovados para uso em pacientes pediátricos; (b) classe não aprovados - medicamentos não aprovados para uso em crianças, que incluiria medicamentos não aprovados para uso como um todo, não aprovados para uso em crianças, contra-indicados para uso em crianças, sem dosagem especificada para crianças $2,3,4,8$; (c) classe não padronizados - medicamentos não padronizados para uso em crianças (em relação à idade do paciente, à dose diária prescrita, ao número de doses prescritas por dia, à via de administração, à apresentação prescrita ou à indicação prescrita), que incluiria medicamentos prescritos de forma diferente daquela orientada nos compêndios de informação farmacológica para idade, dose diária, número de doses por dia, apresentação, via de administração ou indicação para uso em crianças, respectivamente ${ }^{2-4,8}$. As faixas etárias pediátricas consideradas foram: (a) de 1 a 24 meses incompletos; (b) de 2 a 12 anos incompletos; e (c) de 12 a 18 anos incompletos ${ }^{16}$. O escore PIMé um dos índices de gravidade mais utilizados em UTIs pediátricas, considera apenas oito variáveis de fácil aferição no momento da admissão, tendo um bom poder preditivo ${ }^{13}$.

O estudo foi aprovado pela Comissão de Ética e Pesquisa do HCPA, tendo sido isento do termo de consentimento informado. Os autores envolvidos no estudo assinaram termo de compromisso para manutenção do sigilo e para utilização dos dados única e exclusivamente para o fim de publicação científica.

A tabulação dos dados foi realizada em planilha Excel ${ }^{\circledR}$. Foi realizada análise descritiva dos resultados obtidos, bem como comparação de prevalências entre grupos, de acordo 
com as suas características, utilizando o teste do quiquadrado, com um nível de significância de 0,05.

\section{Resultados}

Os dados do estudo foram coletados nos meses de julho e agosto de 2002, em diferentes dias, de seis semanas consecutivas, baseados nas prescrições de 51 pacientes em 54 admissões de UTIP. A idade dos pacientes variou de 1 mês a 13 anos, com mediana de 10,5 meses, sendo $61 \%$ do sexo masculino. Dois terços $(65 \%)$ dos pacientes apresentavam doença prévia e $87 \%$ das admissões ocorreram por motivos clínicos: $57 \%$ por causas respiratórias, $11 \%$ neurológicas, $11 \%$ gastrintestinais, $7 \%$ cardiovasculares, $7 \%$ renais e $7 \%$ por outros motivos.

Foram registrados 747 itens de prescrição, com média de 14 itens por paciente. As prevalências observadas sobre o total de itens de prescrição foram: $40 \%$ para medicamentos aprovados, 10,5\% para medicamentos não aprovados $\mathrm{e}$ $49,5 \%$ para medicamentos não padronizados. Todos os 51 pacientes considerados no estudo tiveram prescrição de pelo menos um medicamento não padronizado, e $88 \%$ deles, pelo menos um medicamento não aprovado.

A distribuição das prevalências de prescrição por faixa etária de pacientes, nas três classes de medicamentos, é mostrada na Tabela 1 , não tendo havido diferença estatística entre elas. Não foi avaliada prescrição em paciente com menos de um mês de idade.

Tabela 1 - Distribuição das prevalências de prescrições por faixa etária de pacientes nas três classes de medicamentos

\begin{tabular}{lccc}
\hline Classe medicamentos & \multicolumn{3}{c}{ Faixa etária } \\
& $\begin{array}{c}\mathbf{1 - 2 4} \text { meses } \\
(\mathbf{n = 3 1})\end{array}$ & $\begin{array}{c}\mathbf{2 - 1 2} \text { anos } \\
(\mathbf{n = 1 7})\end{array}$ & $\begin{array}{c}\boldsymbol{> 1 2} \text { anos } \\
(\mathbf{n = 3})\end{array}$ \\
\hline Aprovados & $204(47,0)$ & $112(44,0)$ & $26(42,5)$ \\
Não aprovados & $55(13,0)$ & $26(10,0)$ & $8(13,0)$ \\
Não padronizados & $173(40,0)$ & $116(46,0)$ & $27(44,5)$ \\
Total de itens prescrição & 432 & 254 & 61 \\
\hline
\end{tabular}

$\left(\chi^{2}=2,65 ; g \mid 4 ; p=0,616\right)$.

$\mathrm{n}$ : refere-se ao número de pacientes em cada faixa etária;

( ) valores percentuais baseados nos itens prescritos por faixa etária de pacientes.

A distribuição das prevalências de prescrição por nível de gravidade de pacientes na admissão, de acordo com as categorias de risco do PIM, nas três classes de medicamentos, é mostrada na Tabela 2, não tendo havido diferença estatística entre elas.

Entre os 747 itens de prescrição, figuravam 131 medicamentos ou apresentações farmacológicas diferentes. Os medicamentos que mais apareceram nas prescrições são mostrados na Tabela 3 .

Os medicamentos não apropriados para crianças são mostrados na Tabela 4 . O uso não padronizado mais prevalente foi em relação à dose, em $50 \%$ dos pacientes, seguido da frequiência de administração, em $28 \%$ dos pacientes.

\section{Discussão}

De acordo com as modernas diretrizes de avaliação de medicamentos, a obtenção de licenciamento para um determinado medicamento ou a obtenção de padronização para uma indicação específica ou para um grupo específico de pacientes exige uma pesquisa demorada e exaustiva ${ }^{1}$. Possivelmente, devido a razões de mercado ou a políticas de investimentos, e, talvez, por não ter sido suficientemente pressionada ou estimulada pelos órgãos oficiais de controle de medicamentos, nas últimas décadas, a indústria farmacêutica não tem estado comprometida na realização de estudos clínicos de eficácia e segurança com grupos pediátricos, para a obtenção de licenciamento de seus produtos para esse segmento.

Poucos são os estudos que avaliam a prevalência de prescrição e uso de medicamentos não apropriados para crianças em UTIP. Jong et al., baseados na avaliação de 2.139 cursos de medicamentos prescritos para 238 crianças de UTIP holandesa, encontraram prevalências de $48 \%$ para medicamentos não aprovados e $18 \%$ para medicamentos não padronizados para crianças ${ }^{2}$. Turner et al., nesse mesmo tipo de população, avaliou 862 itens de prescrição, e demostrou prevalência de $31 \%$ para medicamentos não aprovados ou não padronizados para crianças, indistintamente ${ }^{17}$. O presente estudo avaliou 747 itens de prescrição, e demonstrou prevalência de $10,5 \%$ para medicamentos não aprovados e $49,5 \%$ para medicamentos não padronizados.

Essas altas taxas de prevalência para medicamentos não apropriados para crianças não são exclusividade das UTIPs. Taxas de prevalência muito expressivas também foram observadas em outros locais de assistência pediátrica. McIntyre et al., considerando a prática pediátrica geral, encontraram prevalência de $0,3 \%$ de medicamentos não aprovados e $10,5 \%$ de não padronizados, números que poderiam ser maiores, se fosse verificado o uso não padronizado quanto à indicação ${ }^{4}$. Em ambulatórios pediátricos, o estudo de Chalumeau et al. mostrou $4 \%$ das prescrições com medicamentos não aprovados e $29 \%$ com não padronizados $(65 \%$ para idade, 23\% para indicação, $10 \%$ para dose e $7 \%$ para via), sendo $6 \%$ não padronizado por mais de uma razão ${ }^{7}$. Turner et al. avaliaram 2.013 itens de prescrição em enfermarias clínico-cirúrgicas pediátricas, em 609 pacientes, e observaram uso de $7 \%$ de medicamentos não aprovados e $18 \%$ de não padronizados 5 .

Considerando a faixa etária dos pacientes, no presente estudo, encontramos uma maior prevalência de medica- 
Tabela 2 - Distribuição das prevalências de prescrições por gravidade de pacientes na admissão, de acordo com as categorias de risco do PIM, nas três classes de medicamentos

\begin{tabular}{|c|c|c|c|c|c|}
\hline \multirow[b]{2}{*}{ Classe medicamentos } & \multicolumn{5}{|c|}{ Categoria de risco do PIM } \\
\hline & $\begin{array}{c}<1 \% \\
(n=12)\end{array}$ & $\begin{array}{c}1-4,99 \% \\
(n=17)\end{array}$ & $\begin{array}{c}5-14,99 \% \\
(n=13)\end{array}$ & $\begin{array}{c}15-29,99 \% \\
(n=3)\end{array}$ & $\begin{array}{l}\geq \mathbf{3 0 \%} \\
(\mathrm{n}=5)\end{array}$ \\
\hline Aprovados & $54(42,0)$ & $133(47,0)$ & $90(48,0)$ & $25(49,0)$ & $40(43,0)$ \\
\hline Não aprovados & $23(18,0)$ & $32(11,0)$ & $19(10,0)$ & $2(4,0)$ & $13(14,0)$ \\
\hline Não padronizados & $52(40,5)$ & $120(42,0)$ & $79(42,0)$ & $24(47,0)$ & $41(44,0)$ \\
\hline Totais itens prescrição & 129 & 285 & 188 & 51 & 94 \\
\hline
\end{tabular}

$\left(\chi^{2}=8,87 ; g \mid 8 ; P=0,353\right)$.

PIM: pediatric index of mortality (índice de mortalidade pediátrica), $\mathrm{n}$ : refere-se ao $\mathrm{n}^{\circ}$ de pacientes em cada categoria de risco, ( ) valores percentuais baseados nos itens prescritos por categoria de risco de pacientes.

mentos não apropriados para crianças no grupo etário dos pacientes maiores de 12 anos (61 itens em três pacientes), ainda que o número de pacientes tenha sido pequeno nessa faixa etária. Possivelmente, a ampliação da amostra nesse grupo etário poderia ter alterado essa diferença. McIntyre et al. não encontraram diferença significativa de prevalência de medicamentos não aprovados ou não padronizados entre os grupos etários avaliados ${ }^{4}$. No entanto, no estudo de Chalumeau et al. ${ }^{7}$ ocorreu maior prevalência de medicamentos não padronizados (70\%) em neonatos, provavelmente pela falta de fármacos apropriadamente licenciados para essa faixa etária ou de formulações pediátricas mais flexíveis ${ }^{2}$.

Dos estudos consultados, nenhum classificou a prevalência de prescrição de medicamentos não apropriados para crianças em relação à gravidade dos mesmos. Eventualmente, na UTIP, a gravidade poderia ser considerada uma justificativa para prescrever e usar medicamentos não

Tabela 3 - Medicamentos mais prescritos na UTIP na amostra estudada

\begin{tabular}{lcc}
\hline Medicamento & $\begin{array}{c}\mathbf{n}^{\mathbf{0}} \text { itens } \\
\text { prescrição }\end{array}$ & \%* \\
\hline Midazolam & 56 & 7,5 \\
Paracetamol & 46 & 6,0 \\
Dipirona & 42 & 5,5 \\
Fentanil e Ranitidina & 35 & 4,5 \\
Furosemida, Diazepan e Vancomicina & 25 & 3,5 \\
Hidrato de Cloral & 20 & 2,5 \\
Hidrocortisona, Salbutamol e Quetamina & 17 & 2,0 \\
Dopamina, Cefepime, Metoclopramida e & & \\
Gluconato de cálcio & 16 & 2,0 \\
Fenitoína e Cloreto de potássio & 12 & 1,5 \\
\end{tabular}

* Percentuais sobre $\mathrm{n}^{\circ}$ total de itens prescritos.
Tabela 4 - Medicamentos mais prescritos na UTIP na amostra estudada (por classe)

\begin{tabular}{|c|c|}
\hline $\begin{array}{l}\text { Classe de medicamentos } \\
\text { (prevalência) }\end{array}$ & Medicamentos mais freqüientes \\
\hline Aprovados $(40,0 \%)$ & $\begin{array}{l}\text { Midazolam, Ranitidina, } \\
\text { Vancomicina }\end{array}$ \\
\hline Não aprovados $(10,5 \%)$ & $\begin{array}{l}\text { Dipirona, Ampicilina+Sulbactam, } \\
\text { Omeprazol }\end{array}$ \\
\hline NP para dose $(20,0 \%)$ & Furosemida \\
\hline NP para freqüência $(14,0 \%)$ & Furosemida, Metoclopramida \\
\hline NP para idade $(5,0 \%)$ & Fentanil, Salbutamol \\
\hline NP para apresentação $(5,0 \%)$ & Paracetamol \\
\hline NP para via $(3,0 \%)$ & $\begin{array}{l}\text { Fenitoína, Dexametasona, } \\
\text { Salbutamol }\end{array}$ \\
\hline NP para indicação $(2,5 \%)$ & $\begin{array}{l}\text { Furosemida, Ondansetron, } \\
\text { Óleo mineral tópico }\end{array}$ \\
\hline
\end{tabular}

NP: não padronizados.

aprovados ou não padronizados, invocando a relação risco-benefício. No nosso estudo, embora não se tenha avaliado as causas que pudessem ter levado os profissionais a prescreverem medicamentos não apropriados para crianças, os itens de prescrição de medicamentos não aprovados e não padronizados ficaram igualmente distribuídos entre os diversos grupos de gravidade (de acordo com as categorias de risco do PIM) (Tabela 2), ainda que a amostra de pacientes tenha sido pequena para as duas categorias de maior gravidade (51 itens em 3 pacientes e 94 itens em 5 pacientes, respectivamente).

Estudos de Turner et al. ${ }^{6}$ e de Gill et al. ${ }^{18}$, sobre eventos adversos causados por medicamentos em pacientes pediátricos internados, mostraram que alguns dos medicamentos classificados como não aprovados ou não padronizados no nosso estudo (Tabela 4) poderiam ser os agentes determinantes das reações adversas observadas por eles. 
Pode surprender o fato de medicamentos muito utilizados em prescrições pediátricas terem sido classificados como não padronizados (ex., Paracetamol) ou como não aprovados (ex., Dipirona). No caso do Paracetamol, a formulação prescrita e utilizada na época do estudo tinha apresentação de menor viscosidade, sendo que 1 mililitro correspondia a 30 gotas, enquanto que no produto mais divulgado no mercado 1 mililitro corresponde a 16-20 gotas. Os médicos da unidade desconheciam essa peculiaridade do produto dispensado pela farmácia e prescreviam como se fossem 20 gotas por mililitro, levando à prescrição inadequada, correspondente à dose $50 \%$ inferior à preconizada. Nesse caso, a prescrição do paracetamol foi classificada como não padronizada para apresentação. A Dipirona, medicamento sabidamente não aprovado pela FDA, é freqüentemente prescrita e empregada em pacientes de UTI, pela sua disponibilidade para uso intravenoso, o que favorece sua prescrição em pacientes hospitalizados. Tradicionalmente, tem sido prescrita e utilizada somente em pacientes com febre refratária aos demais antitérmicos, pelo seu maior potencial de efeitos adversos.

No presente estudo, foram detectados outros exemplos de medicamentos não padronizados: (a) em relação à idade, o aerossol de Salbutamol não tem dose estabelecida para uso em crianças até quatro anos de idade, ainda que seja prescrito inclusive para lactentes e crianças pequenas; (b) em relação à dose, o Salbutamol intravenoso não tem dose estabelecida para crianças, ainda que seja uma prática difundida a sua utilização nesse segmento; (c) em relação à freqüência, a Metoclopramida intravenosa é indicada em crianças em dose única, admitindo-se a sua repetição, quando necessário, muito embora ela freqüentemente seja utilizada a intervalos fixos de 6 a 12 horas; (d) em relação à via, a Fenitoína intravenosa só é indicada para tratamento do estado epiléptico, ainda que seja empregada por essa via como terapia anticonvulsivante de manutenção em pacientes com acesso venoso; (e) em relação à indicação, o Ondansetron estaria recomendado apenas para vômitos relacionados à quimioterapia e ao pós-operatório, e não como alternativa para vômitos de outras causas, como tem sido prescrito.

Turner et al. observaram que é mais freqüente a prescrição de medicamentos não padronizados do que não aprovados nos segmentos pediátricos 5 . A maior prevalência de prescrição de medicamentos não padronizados no presente estudo ocorreu em relação à dose, da mesma forma como em outros estudos ${ }^{2,4}$. Esse tipo de prescrição provavelmente ocorreu por falta de informação dos prescritores, o que está de acordo com o estudo de Chalumeau et al. ${ }^{7}$. Imaginase que isso ocorra também em função das apresentações farmacológicas disponíveis, que dificultariam a prescrição de doses precisas.

A prescrição de medicamentos não padronizados para crianças muitas vezes é necessária, aliada ao fato de uma grande quantidade de medicamentos prescritos atualmente não trazerem informações na etiqueta ou na bula sobre doses para crianças. Os prescritores são confrontados com o dilema de prescrever medicamentos sem informação suficiente para dar-lhes segurança, ou deixar seus pacientes sem terapia potencialmente efetiva e, às vezes, salvadora. Nesse contexto, basear-se na literatura médica, na experiência prévia ou na opinião de especialistas pode ser apropriado e dar embasamento aos profissionais que prescrevem. A orientação que acompanha os medicamentos (bula), embora seja importante fonte de informação sobre o medicamento e a sua utilização, não é fator determinante de prescrição adequada, e menos ainda um substituto do julgamento médico ${ }^{2,19}$.

Quando se considera a questão da padronização para a idade, deve-se considerar a enorme variação de peso em crianças, do nascimento aos 12 anos. A prática comum consiste em ajustar a dose de acordo com o tamanho da criança, fazendo com que várias prescrições fiquem fora da orientação recomendada pelas licenças ${ }^{4}$. Além disso, muitas vezes não são encontradas alternativas (medicamentos) licenciadas para algumas situações, o que indica que a origem do problema não é relacionada ao uso inadequado dos medicamentos pelos profissionais, mas a uma avaliação inadequada no processo de registro dos mesmos ${ }^{7}$.

As limitações do presente estudo podem estar relacionadas a eventual vício de aferição, uma vez que as consultas foram baseadas em apenas uma fonte de consulta, internacionalmente aceita, o catálogo USP DI 2001 - Drug Information for the Health Care Professional ${ }^{14}$. Da mesma forma, estudos desse tipo, que fazem alguma forma de controle de qualidade na assistência dada aos pacientes, por mais sigilosos que pretendam ser, nunca passam despercebidos pelos profissionais da unidade. Assim, pode ter havido mudança de comportamento dos prescritores durante a execução do estudo, determinando um vício de seleção, também referido no estudo de Chalumeau et al. ${ }^{7}$. Entretanto, se isso ocorreu, pode ter apenas minimizado a gravidade da situação apresentada. Além disso, o estudo retrata a realidade das doenças de inverno no nosso meio, com avaliação dos itens de prescrição predominantes para uma estação apenas, e não para os das demais épocas do ano.

A FDA regula a produção, aprovação e promoção dos medicamentos, não o uso deles pelos médicos. Novos usos, doses, indicações não são aprovados pela FDA até que seja obtida evidência substancial de segurança e efetividade para uma dada indicação ou grupo etário. Esse processo pode levar anos ou nunca ocorrer, pois, até então, tem havido pouco incentivo para a indústria farmacêutica em conduzir ensaios e submeter informações para novos usos de medicamentos em pacientes pediátricos ${ }^{20}$. As justificativas mais comumente relatadas para a falta de resultados de estudos pediátricos para aprovação de novos medicamentos, ou para novas indicações pediátricas de medicamentos já existentes no mercado têm sido: o excessivo custo dos estudos comparado ao tamanho do mercado potencial entre pacientes pediátricos; a dificuldade de encontrar número suficiente de pacientes para participar de estudos que en- 
contrem significância estatística; o longo tempo que um estudo pediátrico pode levar, prolongando assim o processo de aprovação de um novo medicamento importante; a complexidade dos aspectos éticos associados com a pesquisa em crianças; a escassez de investigadores farmacológicos pediátricos qualificados, e o fato de médicos que tratam crianças prescreverem os medicamentos disponíveis no mercado, sem estarem preocupados na realização de estudos dessa natureza ${ }^{21}$.

No Brasil, a ANVISA (Agência Nacional de Vigilância Sanitária), órgão vinculado ao Ministério da Saúde, tem por finalidade a proteção da saúde da população por intermédio do controle sanitário da produção e da comercialização de produtos e serviços submetidos à vigilância sanitária, incluindo os medicamentos. Uma de suas competências é autorizar o registro de medicamentos no território nacional, baseado em dados e informações de agências reguladoras de reconhecimento internacional ${ }^{15}$.

De acordo com um levantamento realizado pela Consumers 'Association da Grã-Bretanha, poucas indústrias farmacêuticas têm interesse em realizar ensaios clínicos em crianças de modo espontâneo. Mudanças na regulamentação governamental para licenciamento de medicamentos pode ser uma alternativa, para garantir que produtos usados em crianças sejam seguros e efetivos ${ }^{9,22}$. A legislação para medicamentos, originalmente desenhada para proteger os pacientes e os médicos contra o uso não seguro dos mesmos, tornou-se uma barreira para tornar disponíveis medicamentos necessários para esses pacientes ${ }^{2}$.

Concluindo, a alta prevalência de prescrições com medicamentos não apropriados para crianças confirma, também no nosso meio, o uso inadequado e inadvertido de medicamentos não testados ou de apresentações não apropriadas para crianças em UTIP. Isso aponta para a necessidade de estudos bem desenhados e controlados sobre qualidade, eficácia e segurança de medicamentos para uso pediátrico em geral.

A realização de estudos de boa qualidade, avaliando tanto a eficácia quanto a toxicidade de medicamentos em crianças de diferentes faixas etárias e com diferentes estados de doença deveria ser estimulada. Entretanto, o desenvolvimento e a avaliação de medicamentos essenciais para recém-nascidos, lactentes e crianças requer a colaboração entre pediatras, farmacêuticos, indústria farmacêutica e autoridades sanitárias reguladoras.

\section{Referências bibliográficas}

1. Kauffman RE. Essential drugs for infants and children: North American perspective. Pediatrics 1999;104(3 Pt 2):603-5.

2. Jong GW, Vulto AG, de Hoog M, Schimmel KJM, Tibboel D, van den Anker JN. A survey of the use of off-label and unlicensed drugs in a Dutch Children's Hospital. Pediatrics 2001;108: 1089-93.
3. Thompson DF, Heflin NR. Frequency and appropriateness of drug prescribing for unlabeled uses in pediatric patients. Am J Hosp Pharm 1987;44:792-6.

4. McIntyre J, Conroy S, Avery A, Corns H, Choonara I. Unlicensed and off label prescribing of drugs in general practice. Arch Dis Child 2000;83:498-501.

5. Turner S, Longworth A, Nunn AJ, Choonara I. Unlicensed and off label drug use in paediatric wards: prospective study. BMJ 1998;316:343-5.

6. Turner S, Nunn AJ, Fielding K, Choonara I. Adverse drug reactions to unlicensed and off-label drugs on paediatric wards: a prospective study. Acta Paediatr 1999;88:965-8.

7. Chalumeau M, Tréluyer M, Salanave B, Assathiany R, Chéron $\mathrm{G}$, Crocheton N, et al. Off label and unlicensed drug use among French office based pediatricians. Arch Dis Child 2000;83:502-5.

8. Gavrilov V, Lifshitz M, Levy J, Gorodischer R. Unlicensed and off-label medication use in a general pediatrics ambulatory hospital unit in Israel. Isr Med Assoc J 2000;2:595-7.

9. AAP: Committee on Drugs. Guidelines for the ethical conduct of studies to evaluate drugs in pediatric populations. Pediatrics 1995;95:286-344.

10. Meiners MMMA, Bergsten-Mendes G. Prescrição de medicamentos para crianças hospitalizadas: como avaliar a qualidade? Rev Assoc Med Bras 2001;47:332-7.

11. Christensen ML, Helms RA, Chesney RW. Is pediatric labeling really necessary? Pediatrics 1999;104(3 Pt 2):593-7.

12. Wilson JT. An update on the therapeutic orphan. Pediatrics 1999;104(3 Pt 2):585-90.

13. Shann F, Pearson G, Slater A, Wilkinson K. Paediatric index of mortality (PIM): a mortality prediction model for children in intensive care. Intensive Care Med 1997;23:201-7.

14. USP DI 2001. Drug Information for the Health Care Professional. $21^{\text {a }}$ ed. Rockville: United States Pharmacopeial Convention; 2001. vol. 1.

15. Agência Nacional de Vigilância Sanitária [site na Internet]. Disponível: www.anvisa.gov.br. Acessado: 24 de maio de 2003.

16. Autret E. European regulatory authorities and pediatric labeling. Pediatrics 1999;104(3 Pt 2):614-8.

17. Turner S, Gill AM, Nunn AJ, Hewitt B, Choonara I. Use of "offlabel" and unlicensed drugs in paediatric intensive care unit. Lancet 1996;347:549-50.

18. Gill AM, Leach HJ, Hughes J, Barker C, Nunn AJ, Choonara I. Adverse drug reactions in a paediatric intensive care unit. Acta Paediatr 1995;84:438-41.

19. Blumer JL. Off-label uses of drugs in children. Pediatrics 1999;104:598-602.

20. American Academy of Pediatrics. Committee on Drugs. Pediatrics 2002;110:181-3.

21. Cohen SN. The pediatric Pharmacology research unit network and its role in meeting pediatric labeling needs. Pediatrics 1999;104:644-5.

22. KmictowiczZ. Drug industry is unwilling to run trials in children. BMJ 2000;320:1362.

Endereço para correspondência:

Dr. Paulo R.A. Carvalho

Av. Encantado, 249

CEP 90470-420 - Porto Alegre - RS

Tel./Fax: (51) 3330.6334

E-mail: carvalho.conex@uol.com.br 